\title{
Dual NDP52 Function in Persistent CSFV Infection
}

\author{
Shuangqi Fan', Keke $\mathrm{Wu}^{\dagger}$, Chaowei Luo, Xin Li, Mengpo Zhao, Dan Song, \\ Shengming Ma, Erpeng Zhu, Yuming Chen, Hongxing Ding, Lin Yi, Jun Li, \\ Mingqiu Zhao* and Jinding Chen*
}

College of Veterinary Medicine, South China Agricultural University, Guangzhou, China

Viruses have evolved many mechanisms to escape host antiviral responses. Previously, we found that classical swine fever virus (CSFV) infection induces autophagy using the autophagosome as a self-replication site, thereby evading the host immune response and promoting long-term infection. However, the underlying mechanisms used by CSFV to enter autophagosomes and the mechanism by which autophagy promotes viral replication remain unclear. We found that CSFV infection inhibited autophagy receptor nuclear dot protein 52 kDa (NDP52) expression, ubiquitination, and SUMO24 modification. Further analyses revealed that CSFV mediated ubiquitination and SUMOylation of NDP52 via Pten-induced kinase 1 (PINK1)-Parkin. Moreover, NDP52 inhibition also inhibited CSFV replication and the induction of mitophagy marker proteins expression. Inhibition of NDP52 reduced CD63 expression and binding to CSFV E2 protein, which has an essential role in persistent CSFV infection. As NDP52 has a close relationship with the NF-kB innate immunity pathway and plays an important role in the antiviral response, we investigated whether NDP52 inhibited CSFV replication through the release of immune factors and antivirus signals. Our results showed that inhibiting NDP52 boosted interferon and TNF release and promoted NF-кB pathway activation. In summary, we found that NDP52 inhibition not only reduces CSFV binding and entry into autophagic vesicles, but also inhibits CSFV replication by active NF-кB antiviral immune pathways. Our data reveal a novel mechanism by which NDP52, an autophagy receptor, mediates CSFV infection, and provide new avenues for the development of antiviral strategies.

Keywords: classical swine fever virus, NDP52, ubiquitination, CD63, NF- $\mathrm{B}$

\section{INTRODUCTION}

Swine fever, caused by classical swine fever virus (CSFV) infection, is characterized by acute fever and death, and is classified as a Class A infectious disease by the World Organization for Animal Health (OIE) (Paton and Greiser-Wilke, 2003). CSFV belongs to the genus Pestivirus within the family Flaviviridae. The virus has a small, enveloped, single-stranded, positive-sense $12.3 \mathrm{~kb}$ RNA genome with a long, open reading frame that encodes a 3898 amino acid polypeptide (Becher et al., 2003). Co-and post-translational processing of the polypeptide by cellular and viral proteases yields 12 cleavage products, including four structural proteins (C, Erns, E1, and E2) and eight nonstructural proteins (N ${ }^{\text {pro }}$, p7, NS2, NS3, NS4A, NS4B, NS5A, and NS5B) (Heinz-Jurgen et al., 1991). 
CSFV can infect several cells types, including immune cells, leading to cellular immunosuppression (Fan et al., 2018). However, CSFV infection does not cause typical pathological changes, and the underlying infection mechanisms remain unclear (Bensaude, 2004; Johns et al., 2009).

Macroautophagy, hereafter referred to as autophagy, is an internal balancing mechanism for maintaining homeostasis in eukaryotic cells. After receiving an autophagy induction signal, such as pathogen infection (Deretic et al., 2013), starvation (Tattoli et al., 2012), growth factor withdrawal (Lum et al., 2005), endoplasmic reticulum (ER) stress (Ciechomska et al., 2013), or oxidative stress (Scherz-Shouval et al., 2007), the cell forms a small liposome-like membrane structure in the cytosol, which expands to form a bowl-like structure consisting of two layers of lipid bilayers that can be observed under electron microscopy. The bowl structure is called a phagophore. Many components in the cytoplasm, including protein aggregates, damaged organelles, and foreign invading pathogens, are wrapped in vesicles and then closed into a closed spherical autophagosome (Høyer-Hansen and Jäättelä, 2008). Microtubule-associated protein 1 light chain 3 (MAP1LC3 or simply LC3), consisting of the interconvertible forms LC3-I and LC3-II, is involved in the formation of autophagosome membranes. Early pro-LC3 cleavage by ATG4 exposes the C-terminal glycine to form the cytosolic soluble form LC3-I, which is modified by ubiquitination and coupled with the substrate $\mathrm{PE}$ on the surface of the autophagosome membrane under the action of the E1-like enzyme ATG7, the E2-like enzyme ATG3, and the E3-like enzyme ATG5-ATG12ATG16L complex to form the membrane-bound form LC3-II. After autophagosome formation, this fuses with lysosomes to form autolysosomes. Under the action of various hydrolases, the substrate in the autophagosomes is degraded (Bizargity and Schröppel, 2014). Autophagy is a way for cells respond to unfavorable environmental factors. Many RNA viruses, such as enteroviruses, hepatitis $\mathrm{C}$ virus (HCV), and CSFV, circumvent and utilize host autophagic machinery to promote viral propagation (Pei et al., 2013; Mohamud and Luo, 2018; Wang and $\mathrm{Ou}, 2018$ ). During CSFV infection, the viral proteins NS5A and E2 colocalize with the autophagy marker CD63 on autophagosome-like vesicle membranes. Moreover, CSFV infection can use mitophagy to inhibit cell apoptosis to create a persistent environment for viral infection (Pei et al., 2016; Gou et al., 2017). However, the mechanisms underlying CSFVautophagosome entry are unclear.

Autophagy was initially considered to be non-selective, but recent studies have found that autophagy can also be selective. The most important feature of the selective autophagy pathway is the involvement of autophagy receptors that recognize and transport autophagic substrates, thereby regulating autophagy substrate degradation under very precise dynamic control (Lazarou et al., 2015). These autophagy receptors contain a conserved LC3-interacting region (LIR) domain, which binds to Atg8 molecules on autophagosomes and mediate autophagy degradation (Zaffagnini and Martens, 2016). Ubiquitin acts as a signaling molecule, inducing polyubiquitination of autophagy substrates (Kocaturk and Gozuacik, 2018). Autophagy receptor proteins recognize and bind autophagic substrates in a UBA domain-dependent or independent manner. The LIR is anchored to the autophagosome membrane, followed by autophagosome fusion, lysosome fusion, and substrate degradation in lysosomes (cargo recognition and trafficking in selective autophagy) (Shaid et al., 2012; Nakamura and Yoshimori, 2017). In addition to the autophagy receptor functions, the protein itself is also ubiquitinated. Hou et al. found that the ubiquitin ligase HACE1 with tumor suppressor activity binds to the OPTN protein and catalyzes the polyubiquitination of OPTN. OPTN is modified by HACE1 with a K48-linked polyubiquitin chain, followed by autophagy-lysosomal pathway degradation. HACE1 mediates the modification of the 193th lysine of OPTN by a ubiquitin chain, which specifically interacts with the UBA domain of p62 to activate autophagy. Clearly, ubiquitinated OPTN interacts with p62 to increase the efficiency of autophagy receptor-mediated transport of substrates to autophagic vacuoles, significantly increasing autophagic flux and thereby more effectively recruiting and transporting autophagy substrates (Liu et al., 2014).

It is well known that NF- $\mathrm{B}$ plays a key role in regulating the immune response to infection. It participates in the inflammatory response and immune response and regulates apoptosis and the stress response (Liu et al., 2017). There are three main signal transduction pathways that activate NF-кB: the classical pathway, bypass pathway, and atypical pathway (Oeckinghaus and Ghosh, 2009). Several proteins encoded by NF- $\kappa \mathrm{B}$ target genes are involved in the activation of immune and inflammatory responses. NF- $\kappa \mathrm{B}$ activation during viral infection is interpreted as a host protective response to viral pathogens (Schmitz et al., 2014). Type I interferons (IFNs) also play an important role in the immune response to viruses. The production of type I IFN in immune cells is mediated by pattern recognition receptors in the host cell (Ivashkiv and Donlin, 2013). There are four main ways to induce the production of type I IFN: (1) DNA virus activates the second messenger cGAMP (cyclic GMPAMP) induction pathway; (2) RNA virus activates the RIGI-like receptor (RLR) induction pathway; (3) the Toll-like receptors TLR3 and TLR4 activate the adaptor protein TRIF induction pathway; and (4) TLR7/TLR8 and TLR9 activate the transcription factor IRF7 induction pathway (Majzoub et al., 2019). Interestingly, CSFV replication in cells suppresses type I IFN-inducible antiviral activity and apoptosis by interfering with IFN production, resulting in the persistent survival of CSFV in host cells in vitro.

Nuclear dot protein $52 \mathrm{kDa}$ (NDP52), also called CALCOCO2, has been well studied in xenophagy and primary adaptation to Parkin-mediated mitophagy (Sharma et al., 2018; Ravenhill et al., 2019). Pten-induced kinase 1 (PINK1) can promote NDP52 recruitment and ubiquitination. Further, the xenophagy kinase TBK1 forms a complex with NDP52, thus promoting xenophagy (Fu et al., 2018). In Salmonella typhimurium infection, NDP52 promotes pathogen-containing autophagosome maturation and independently regulates targeting of bacteria to mature autophagosomes (Verlhac et al., 2015a). Further studies have found that Rab35 GTPase and myosin VI play important roles in 
NDP52-microorganism binding and autophagosome maturation (Minowa-Nozawa et al., 2017). During viral infection, NDP52 not only interacts with viral proteins, but also activates RIG-I and NF- $\kappa$ B signaling pathways to exert antiviral effects (Jin et al., 2017). Many studies have shown that NDP52 has a negative regulatory effect on the $\mathrm{NF}-\kappa \mathrm{B}$ pathway. Moreover, in CVB virus infection, CALCOCO2, but not SQSTM1, suppresses antiviral type I IFN signaling by promoting autophagy-mediated degradation of the mitochondrial antiviral signaling (MAVS) protein (Mohamud et al., 2018). Therefore, we sought to explore the regulatory effect of NDP52 on NF- $\kappa$ B and IFN in swine fever virus infection and hypothesized that it plays an important role in CSFV infection.

Here, we found that CSFV infection activates the PINK1Parkin pathway, resulting in NDP52 SUMOylation, which inhibits NDP52 and permits CSFV replication. Further, NDP52 colocalizes with viral protein E2, thereby inhibiting CD63 expression, promoting CSFV binding by NDP52 and increasing cytokine release and NF- $\kappa \mathrm{B}$ signaling activation.

\section{MATERIALS AND METHODS}

\section{Cells, Viruses, and Virus Titration Assays}

The swine kidney cell line PK-15 (ATCC, CCL-33) was grown in Dulbecco's modified Eagle's medium (DMEM) supplemented with $10 \%$ fetal bovine serum (FBS) at $37^{\circ} \mathrm{C}$ in a $5 \% \mathrm{CO}_{2}$ incubator. The CSFV strain (Shimen) used in the study was propagated in PK-15 cells. Viral titers in CSFV-infected cell culture media were determined as previously described (Hongchao et al., 2017). Briefly, cells cultivated in 96-well plates were inoculated with 10 -fold serial dilutions of virus and incubated at $37^{\circ} \mathrm{C}$ for 3 days. Cells were fixed with $80 \%$ acetone at $-20^{\circ} \mathrm{C}$ for $30 \mathrm{~min}$, and viruses were detected by immunofluorescence assay using mouse anti-CSFV E2 antibody and FITC-conjugated goat anti-mouse secondary antibody. Viral titers are expressed as 50\% tissue culture infective doses (TCID50)/0.1 ml.

\section{Reagents and Antibodies}

The chemical reagents MG-132 (M8699) and BAY (11-7082, S2913) were purchased from Sigma-Aldrich and Selleck. The following primary antibodies were used in this study: rabbit polyclonal anti-PARK2 (Abnova, PAB0714), rabbit polyclonal anti-LC3B (Cell Signaling, 2775), rabbit polyclonal anti-MFN2 (Santa Cruz, sc-50331), goat polyclonal anti-VDAC1 (Santa Cruz, sc-32063), goat polyclonal anti-TOM20 (Santa Cruz, sc-11021), mouse monoclonal anti-Beclin-1 (Cell Signaling, 2A4), rabbit polyclonal anti-CD63 (Beyotime, AF1471), mouse monoclonal anti-Ub(A-5) (Santa Cruz, sc-166553), mouse monoclonal antiCSFV E2 (JBT, 9011), mouse monoclonal anti-IkBa (Cell Signaling, 112B2), mouse monoclonal anti-kB-Ras2 (Santa Cruz, sc-374311), rabbit monoclonal anti-P65 (Santa Cruz, sc-AF1870), mouse monoclonal anti-GAPDH (Beyotime, AG019), mouse monoclonal anti-tubulin (Beyotime, AT819), normal rabbit IgG (Beyotime, A7016), and normal goat IgG (Beyotime, A7007). The polyclonal anti-CSFV Npro was kindly provided by Dr. Xinglong
$\mathrm{Yu}$ (Veterinary Department, Hunan Agricultural University, China). The secondary antibodies used for immunofluorescence were Alexa Fluor 350 goat anti-mouse IgG (Beyotime, A0412), Alexa Fluor 488 goat anti-mouse IgG (Beyotime, A0428), and Alexa Fluor 647 goat anti-rabbit IgG (Beyotime, A0468). The secondary antibodies used for immunoblotting analysis were HRP-conjugated goat anti-mouse IgG (Bioworld Technology, BS12478), HRP-conjugated goat anti-rabbit IgG (Bioworld Technology, BS13278), and HRP-conjugated rabbit anti-goat IgG (Bioworld Technology, BS30503).

\section{Plasmids and RNA Interference}

The EGFP-LC3 plasmid was prepared in our laboratory. Plasmid pAT016 (p-mito-mRFP-EGFP) was a kind gift from Dr. Andreas Till (University of California, United States). Parkin-targeting shRNAs and scrambled shRNA were obtained from Cyagen. Small interfering RNAs (siRNAs) for NDP52 were synthesized by Sangon Biotech. The shRNA and siRNA sequences are listed in Table 1. PK-15 cells grown to $60 \%$ confluence in six-well cell culture plates were transfected with siRNA and shRNA using Lipofectamine 3000 reagent (Thermo Fisher, L3000015). Targeted protein knockdown was evaluated by western blotting.

\section{Virus Infection}

Twenty-four hours after siNC or siRNA transfection, cells were infected with CSFV at a multiplicity of infection (MOI) of 0.1 . Two hours later, the viral inoculum was removed and the infected cells were washed twice with phosphate-buffered saline (PBS) ( $\mathrm{pH}$ 7.4). DMEM containing 2\% FBS was then added to each culture. At various time points post-infection, cell-free culture supernatants and cell lysates were harvested and stored at $-80^{\circ} \mathrm{C}$ until use.

\section{Immunoprecipitation}

For immunoprecipitation analysis, PK-15cells were infected with CSFV at an MOI of 0.1 for $24 \mathrm{~h}$. SUMOylated NDP52 and ubiquitinated Parkin or NDP52 from whole cell lysates (WCL) were incubated on ice with IP lysis buffer (Beyotime, P0013) containing 1 mM PMSF (Beyotime, ST506) for $10 \mathrm{~min}$. The precipitates were removed by centrifugation at $14,000 \times g$ for $10 \mathrm{~min}$ at $4^{\circ} \mathrm{C}$. The supernatant was immunoprecipitated with the appropriate antibodies (antiParkin or anti-NDP52) and protein A + G Sepharose (7sea

TABLE 1 | shRNA and siRNA sequences of targeted genes.

\begin{tabular}{ll}
\hline Gene & Sequence $\left(\mathbf{5}^{\prime} \sim \mathbf{3}^{\prime}\right)$ \\
\hline shParkin & GCATCACCTGTACGGACATTCTCTTGAAATGTCCGTACAG \\
strambled & GCGCGCTTGGTAGGATCGTTCAAGAGACGAATCCTACAAA \\
& GCGCGC \\
siNDP52 & Sense: GCAGGAAGUCCAGUUCAAATT \\
& Antisense: UUUGAACUGGACUUCCUGCTT \\
siNC & Sense: UUCUCCGACGUGUCACGUTT \\
& Antisense: ACGUGACACGUUCGGAGAATT
\end{tabular}


biotech, P001-2). The immunoprecipitated proteins were then analyzed by western blotting with SUMO or ubiquitin antibodies.

\section{Quantitative Real-Time RT-PCR (qPCR)}

For targeted gene expression analysis, total RNA was prepared using a total RNA Kit I (Omega, R6834-01). Complementary DNA (cDNA) was synthesized using PrimeScript RT Master Mix (Takara, RR036A). Real-time qPCR was performed using SYBR Premix Ex Taq II (Takara, RR820A) using an iQ5 iCycler detection system (Bio-Rad, United States). Relative mRNA expression was assessed using the $2^{-\Delta \Delta C t}$ method and normalized to the housekeeping gene GAPDH. The primers used are described in Table 2. For virus copy detection, viral RNA was extracted using a MiniBEST Viral RNA/DNA Extraction Kit Ver.5.0 (Takara, 9766) and reversetranscribed using PrimeScript RT Master Mix (Perfect Real Time; Takara, RR036A). The resulting cDNA was then amplified using SYBR Premix Ex Taq (Tli RNaseH Plus; Takara, RR420B) and an iQ5 iCycler detection system (Bio-Rad, United States). Primer sequences targeting the CSFV NS5B gene were: CSFV1: CCTGAGGACCAAACACATGTTG; CSFV2: TGGTGGAAGTTGGTTGTGTCTG. Viral copy number was calculated using a standard curve from a recombinant plasmid containing the CSFV NS5B gene.

\section{Western Blot Analysis}

After treatment, cells were washed with cold PBS and incubated on ice with RIPA lysis buffer (Beyotime, P0013B) supplemented with $1 \mathrm{mM}$ PMSF (Beyotime, ST506) for $10 \mathrm{~min}$. Cell lysates were centrifuged at $14,500 \times g$ for $20 \mathrm{~min}$ at $4^{\circ} \mathrm{C}$. Protein concentration was determined using a BCA protein assay kit (Beyotime, P0012). Samples with equal protein amounts were diluted in $5 \times$ SDS-PAGE loading buffer and boiled for $5 \mathrm{~min}$. Proteins $(20 \mathrm{mg})$ were separated by SDS-PAGE and transferred onto polyvinylidene fluoride membranes (Beyotime, FFP30). After blocking with PBS containing 2\% non-fat milk powder and $0.05 \%$ Tween 20 (Sigma-Aldrich, P2287) for $2 \mathrm{~h}$ at $25^{\circ} \mathrm{C}$, the membrane was incubated with primary antibodies overnight at $4^{\circ} \mathrm{C}$. Then, membranes were incubated with corresponding HRP-conjugated secondary antibodies at $37^{\circ} \mathrm{C}$ for $2 \mathrm{~h}$ at appropriate dilutions. The protein bands were visualized using an ECL Plus kit

TABLE 2 | Primers used in this study.

\begin{tabular}{lll}
\hline Gene & Sequence $\left(\mathbf{5}^{\prime} \sim \mathbf{3}^{\prime}\right)$ & GenBank \\
\hline NDP52 & F: CGGATTCCATGAGGGGCGGGCCCCG & XM003131552.4 \\
& R: GGGGTACCTCACAGGTCCTTCAGATCCTT & \\
IFNA & F: CTCAGCCAGGACAGAAGCA & NM_214393.1 \\
& R: TCACAGCCCAGAGAGCAGA & NM_001003923.1 \\
IFNB1 & F: TCGCTCTCCTGATGTGTTCTC & \\
& R: AAATTGTGCTCCTTGTTGGT & EU682384 \\
TNF & F: TGGCCCAAGGACTCAGATCAT & NM_001206359.1 \\
& R: TCGGCTTGACATTGGCTACA & \\
GAPDH & F: TGGAGTCCACTGGTGTCTTCAC &
\end{tabular}

(Beyotime, P0018). Blots were imaged with a CanoScan LiDE 100 scanner (Canon, Japan) and quantified with Image Pro Plus 6.0 software.

\section{Confocal Microscopy}

Cells were grown in $35 \mathrm{~mm}$ glass-bottom petri dishes (NEST, GBD-35-20). The indicated interfering RNA was transfected at various time points. Cells were washed with PBS, fixed with $4 \%$ paraformaldehyde for $30 \mathrm{~min}$, and washed with $0.2 \%$ Triton X100 (Sigma-Aldrich, T8787) in PBS for $10 \mathrm{~min}$. The cells were blocked in PBS containing 5\% bovine serum albumin (BSA; Beyotime, ST023) for $30 \mathrm{~min}$. Next, the cells were stained with primary antibodies and appropriate secondary antibodies for $1 \mathrm{~h}$ at $37^{\circ} \mathrm{C}$. Wherever indicated, nuclei were stained with DAPI (Beyotime). Fluorescence was visualized with a TCS SP2 confocal fluorescence microscope (Leica).

\section{Cell Viability Assay}

Cell viability was detected by the CCK8 assay according to the manufacturer's instructions (Dojindo, CK04). In brief, PK-15 cells were cultivated in 96-well plates at a density of $1 \times 10^{4}$ cells per well and cultured for $24 \mathrm{~h}$ at $37^{\circ} \mathrm{C}$. The cells were transfected with siNDP52, siNC, shParkin or non-targeting shRNA using Lipofectamine 3000 reagent. After $48 \mathrm{~h}$, the medium was replaced with $100 \mu \mathrm{l}$ of fresh medium containing $10 \mu \mathrm{l}$ of CCK8. The cells were further cultured for $1 \mathrm{~h}$ at $37^{\circ} \mathrm{C}$, and the optical density was measured at $570 \mathrm{~nm}$ using a model 680 microplate reader (Bio-Rad).

\section{Statistical Analysis}

Statistical analysis was performed with unpaired Student's $t$-tests, as implemented in GraphPad Prism 5 software (mean $\pm \mathrm{SD}$; $\left.n=3 ;{ }^{*} P<0.05 ;{ }^{* *} P<0.01 ;{ }^{* * *} P<0.001 ;{ }^{*} P>0.05\right)$.

\section{RESULTS}

\section{CSFV Infection Inhibits NDP52 (CALCOCO2) Ubiquitination and SUMOylation}

To explore whether CSFV infection affects NDP52, we first evaluated changes in NDP52 mRNA and protein expression after CSFV infection. PK-15 cells were tested at several time points post-CSFV infection. NDP52 mRNA and protein expression was decreased by CSFV infection at 24, 36, and $48 \mathrm{~h}$ post-infection (hpi) (Figures 1A,B). These results suggest that CSFV infection has a negative effect on the expression of NDP52. Several reports indicate that autophagy receptors undergo self-modification (Liu et al., 2014). Therefore, we examined NDP52 ubiquitination and SUMOylation after CSFV infection. CSFV-infected cell lysate was immunoprecipitated with an NDP52-specific antibody and immunoblotted with SUMO2-4 or Ub(A-5) antibody. These results showed that NDP52 ubiquitination and SUMOylation were decreased in CSFV-infected cells compared to that in uninfected PK-15 cells (Figures 1C,D). The above results indicate 


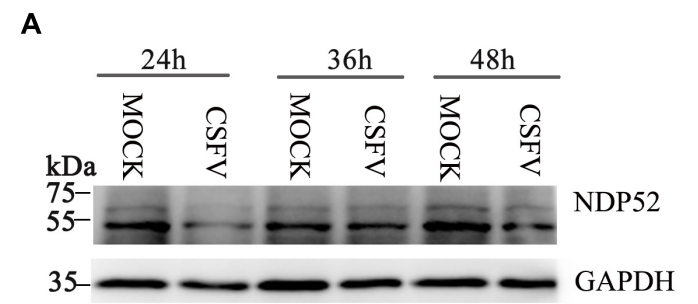

C

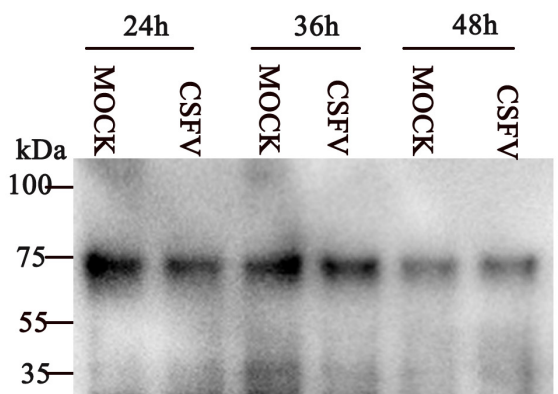

IP:NDP52

IB:SUMO2-4

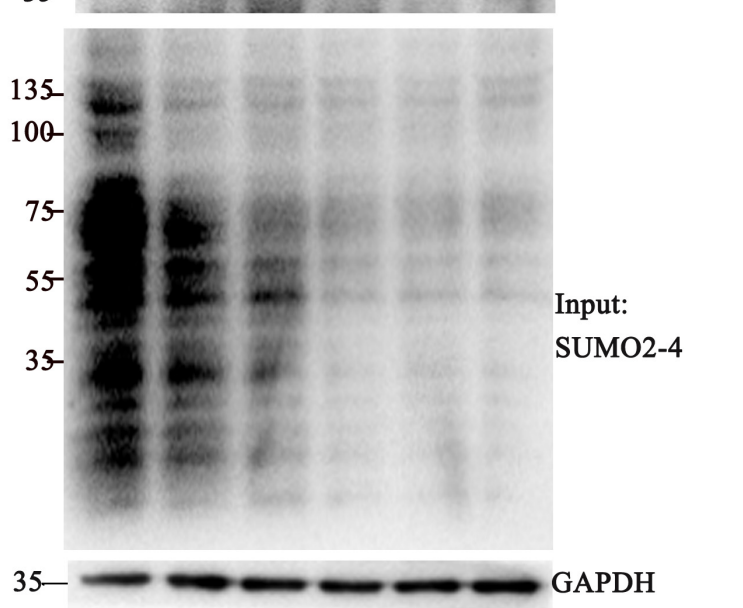

B

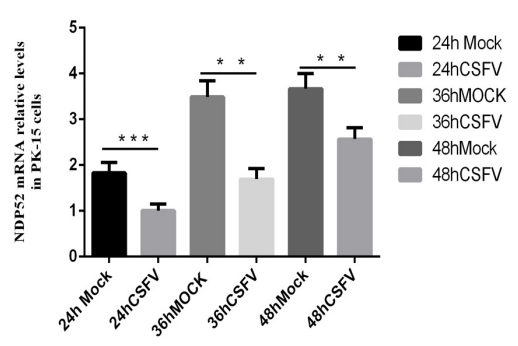

D

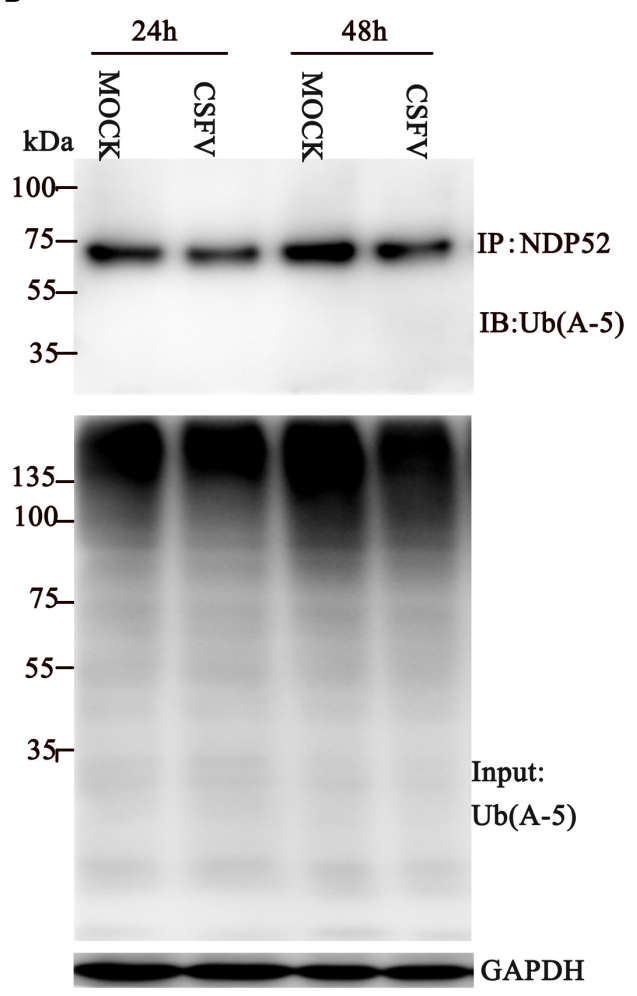

FIGURE 1 | Classical swine fever virus (CSFV) inhibits NDP52 (CALCOCO2) ubiquitination and SUMOylation. (A) PK-15 cells were infected with CSFV (MOI = 0.1$)$ or un-infected (MOCK) at the indicated time points. CALCOCO2, N ${ }^{\text {pro }}$, and GAPDH levels were analyzed by Western blot. (B) After CSFV infection, CALCOCO2 mRNA was detected by qRT-PCR. The data represent the mean \pm SD of three independent experiments. Data were tested by one-way ANOVA with Fisher's least significant difference (LSD) post hoc correction. ${ }^{*} P<0.05$; ${ }^{* *} P<0.01$; ${ }^{* * *} P<0.001$; \#P $>0.05$. (C) PK-15 cells were infected with CSFV. NDP52 SUMOylation was analyzed by immunoblot using anti-SUMO2-4 antibody. SUMO2-4 expression in CSFV-infected cells was used as the input control. (D) Ubiquitinated NDP52 was immunoblotted with anti-Ub(A-5) antibody. Ub(A-5) expression in CSFV-infected cells was used as the input control. GAPDH was used as the internal loading control.

that CSFV infection not only inhibits the expression of NDP52, but also mediates the protein modification of NDP52.

\section{CSFV Infection Promotes Ubiquitination of Parkin and Mediates NDP52 Modification via PINK1-Parkin}

Several reports suggest that NDP52 is closely related to the PINK1-Parkin pathway (Heo et al., 2015). To verify whether CSFV infection affects NDP52 modification via the PINK1-Parkin pathway, we first examined Parkin expression and ubiquitination at $24 \mathrm{~h}$ post-CSFV infection. We observed that CSFV infection promotes Parkin2 protein expression and ubiquitination (Figures 2A,B). The shParkin interference plasmid (Table 1) was applied to PK-15 cells for $24 \mathrm{~h}$. Then, cells were infected with CSFV at an MOI of 0.1. Cell lysate was immunoprecipitated with anti-NDP52 antibody and immunoblotted with SUMO24 antibody or $\mathrm{Ub}(\mathrm{A}-5)$ antibody. The results showed that ubiquitinated and SUMOylation NDP52 were significantly increased with shParkin, suggesting that Parkin plays a key role in NDP52 protein modification (Figure 2C). Thus, CSFV can activate the PINK1-Parkin pathway and promote the expression 
A

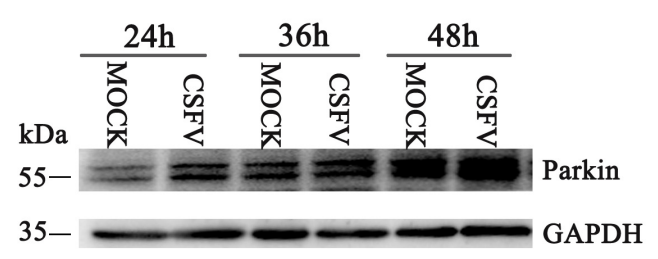

B $\mathrm{kDa}$ IP: anti-Parkin
IB: anti-Ub(A-5)

55
C

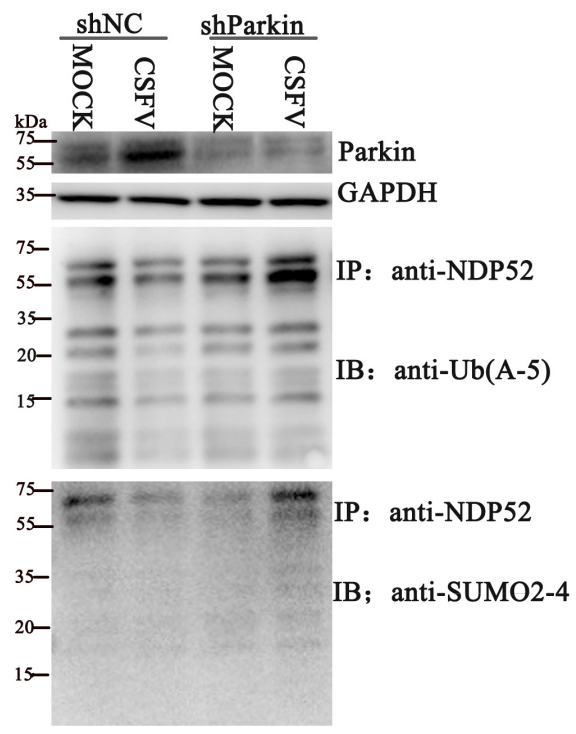

FIGURE 2 | CSFV infection promotes ubiquitination of Parkin2 and mediates NDP52 modification via the PINK1-Parkin pathway. (A) PK-15 cells were treated as in Figure1 A. Western blotting was used to examine Parkin2, Noro and GAPDH protein expression 24, 36, and $48 \mathrm{~h}$ after CSFV infection. (B) PK-15 cells were infected with CSFV (MOI = 0.1) for $24 \mathrm{~h}$. Cell lysates were immunoprecipitated with an anti-Parkin antibody and assayed for Parkin2 with anti-Ub(A-5) antibody. (C) PK-15 cells were infected with CSFV (MOI $=0.1)$ for $24 \mathrm{~h}$, followed by shParkin2 or shNC plasmid $(\mathrm{MOI}=10)$ for $24 \mathrm{~h}$. After immunoprecipitation with an anti-NDP52 antibody, NDP52 ubiquitination and SUMOylation were blotted with anti-Ub-5a and anti-SUMO2-4 antibodies.

of Parkin. After inhibition of Parkin, the protein modification of NDP52 induced by CSFV is attenuated. These results show that CSFV infection promotes the ubiquitination of Parkin and mediates NDP52 modification via the PINK1-Parkin pathway.

\section{NDP52 Inhibition Decreases CSFV Replication}

To assess the functional effects of NDP52 on CSFV infection, we performed RNA knockdown experiments to silence endogenous NDP52 expression in CSFV-infected PK-15 cells. As shown in Figure 3A, the silencing effect on NDP52 expression was verified by western blotting. Decreased expression of CSFV $\mathrm{N}^{\text {pro }}$ protein in NDP52-silenced cells suggested that CSFV replication is promoted by NDP52. To verify this finding, we analyzed CSFV replication after treatment with NDP52 siRNA, by measuring viral titers and RNA copy numbers. These results indicated that NDP52 silencing decreased viral replication in PK-15 cells (Figures 3B,C), demonstrating a positive role for NDP52 in CSFV replication. Moreover, we found that CSFV E2 protein and NDP52 colocalize to the cytoplasm by confocal microscopy (Figure 3D). The above results indicated that NDP52 promotes replication of CSFV and can colocalize with CSFV structural proteins.

\section{NDP52 Inhibition Reduces Autophagy Related Proteins Expression, but Decreases Ubiquitin and LC3 Binding}

Several studies show that NDP52 is essential for autophagosome maturation (Tumbarello et al., 2012; Verlhac et al., 2015b). To investigate the role of NDP52 in CSFV-mediated autophagy, we examined the effect of NDP52 on autophagy and mitophagy. We assessed expression of the autophagy marker LC3 by 


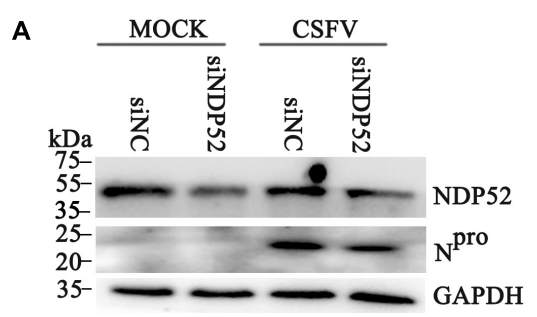

B

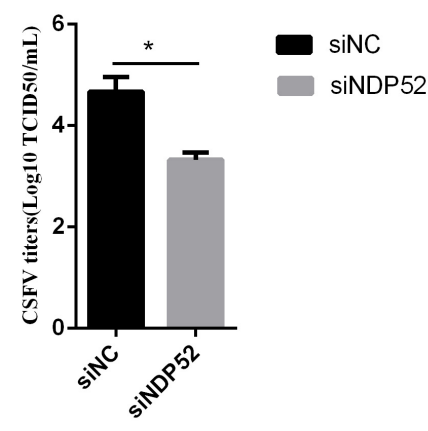

48 Hours-post infection
C

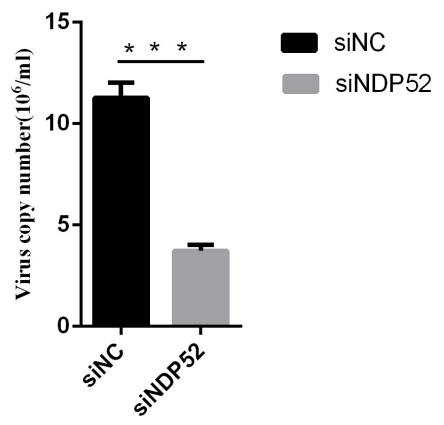

48 Hours-post infection

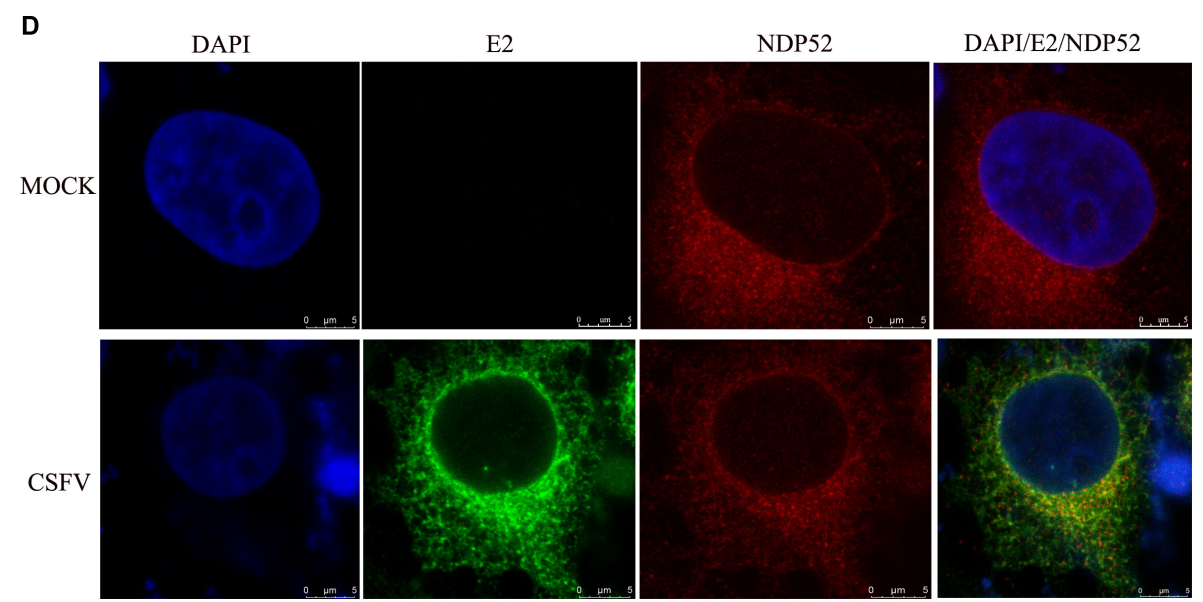

FIGURE 3 | NDP52 inhibition reduces CSFV replication. (A) PK-15 cells were mock-infected or infected with CSFV (MOI $=0.1)$ after transfection with siNDP52 for $24 \mathrm{~h}$. NDP52 expression was analyzed by Western blotting at 24 hpi. CSFV infection was verified by immunoblotting with anti-CSFV Noro antibody. GAPDH was used as an internal loading control. (B) Statistical analysis of the effect of NDP52 siRNA transfection on the viral copy numbers in CSFV-infected cells. PK-15 cells were transfected with scrambled or NDP52 siRNA for $24 \mathrm{~h}$, followed by mock infection and CSFV infection $(\mathrm{MOI}=0.1)$. At 48 hpi, CSFV RNA levels were analyzed by real-time qRT-PCR (mean $\pm \mathrm{SD} ; n=3 ;{ }^{*} P<0.05 ;{ }^{* *} P<0.01 ;{ }^{* * *} P<0.001 ;{ }^{\#} P>0.05$ ). $P$-values were calculated using an unpaired Student's $t$-test.

(C) Statistical analysis of the effect of NDP52 siRNA transfection on virus titers in CSFV-infected cells. PK-15 cells were transfected with scrambled or NDP52 siRNA for $24 \mathrm{~h}$, followed by mock infection and CSFV infection (MOI = 0.1). At $48 \mathrm{hpi}$, CSFV titers were analyzed (mean $\pm \mathrm{SD} ; n=3 ;{ }^{*} P<0.05 ;{ }^{* *} P<0.01 ;{ }^{* * *} P<0.001$; ${ }^{\#} P$ > 0.05). $P$-values were calculated using an unpaired Student's $t$-test. (D) PK-15 cells were mock-infected or infected with CSFV (MOI $\left.=0.1\right)$, after $24 \mathrm{~h}$ infection, cells were immunostained with antibodies against CSFV E2 (green) and NDP52 (red). In the merged images, protein colocalization is displayed as yellow.

transfecting the plasmid of GFP-LC3 and observed by fluorescence microscopy (Figure $\mathbf{4 A}$ ). We also used the plasmid of GFP-RFP-LC3 to detect its distribution in cells. The results showed that LC3B in the cytoplasm was reduced and the yellow spots more than red spots in NDP52-inhibited cells. Thus, inhibition of NDP52 reduced LC3 expression and the normal fusion of autophagosomes with lysosomes. We also assessed the mitochondrial autophagy marker Tom20 using laser confocal microscopy and found that inhibition of NDP52 enhanced Tom20 expression (Figure 4B), suggesting positive regulation of autophagy by NDP52. At the same time, we detected expression of cellular and mitochondrial autophagy markers LC3B and Beclin-1 expression decreased, while VDAC1 and Tom 20 expression increased (Figure 4C), indicating that 


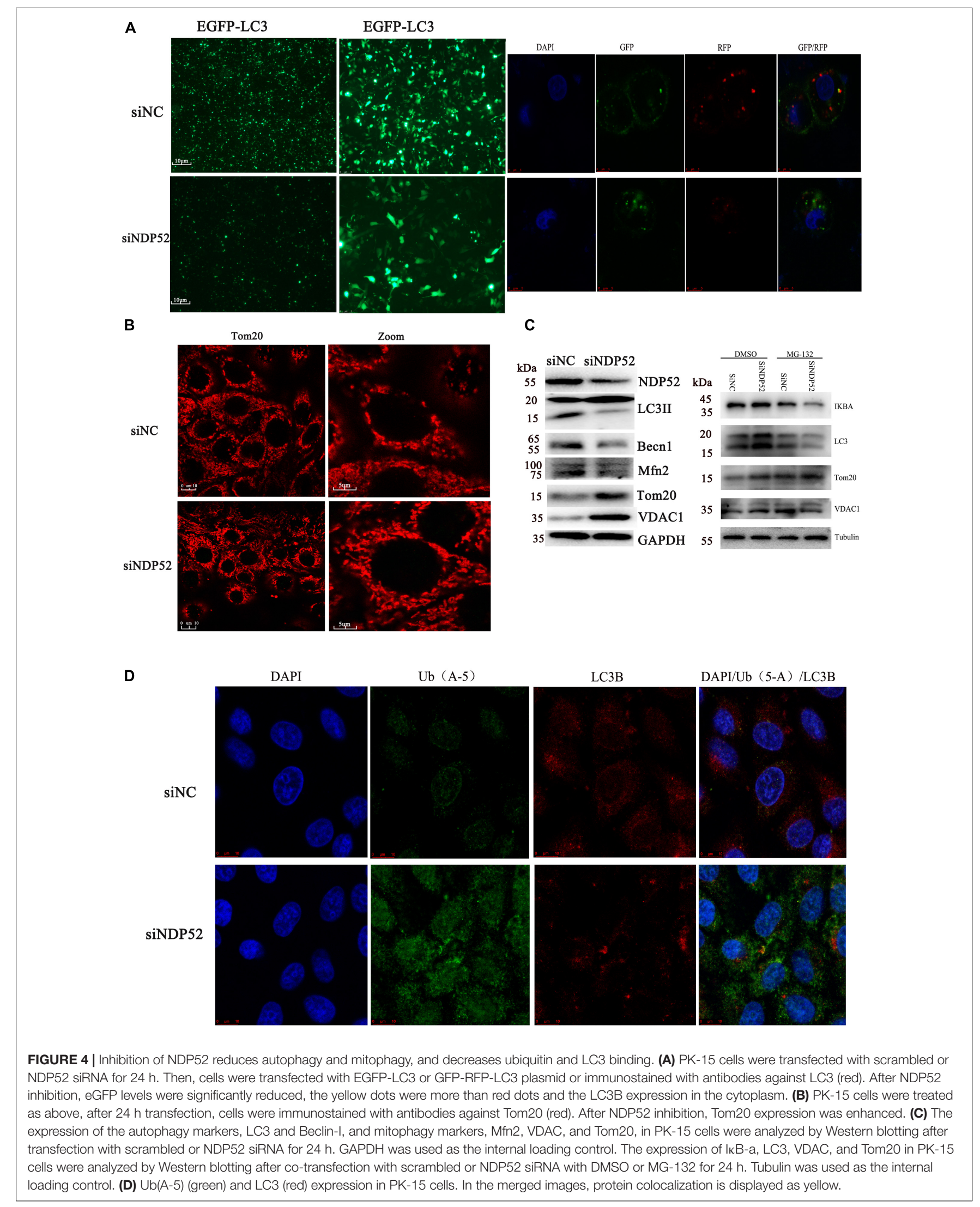




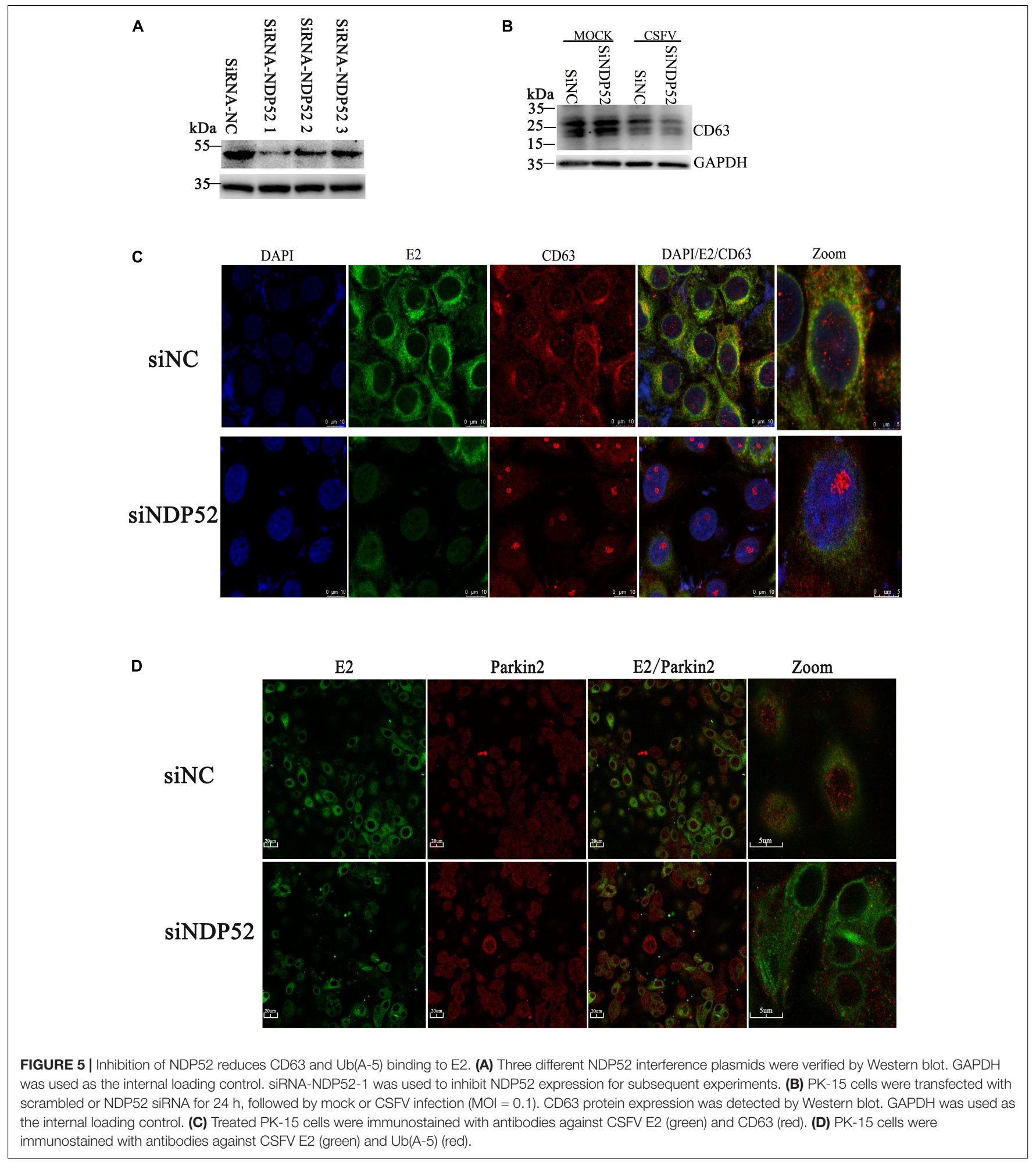

inhibition of NDP52 has an inhibitory effect on the expression of autophagy proteins. To explore the role of ubiquitin in this process, we co-transfected NDP52 interfering RNA with MG-132 $(5 \mu \mathrm{M})$, a ubiquitin-proteasome system inhibitor. We found that in the cells treated with MG-132, the effect of SiNDP52 on the autophagy-related protein disappeared, indicated that ubiquitin-proteasome system affects the effect of NDP52 on autophagy. In selective autophagy, the function of NDP52 is closely related to LC3 and ubiquitin, so we examined the expression and colocalization of LC3 and $\mathrm{Ub}(\mathrm{A}-5)$ in cells. 


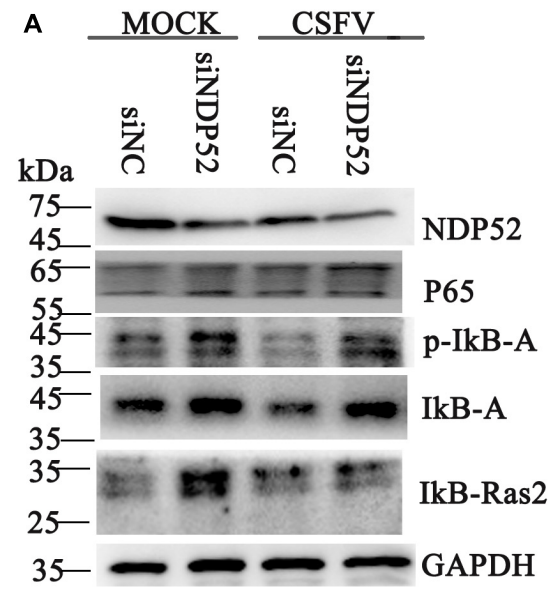

C

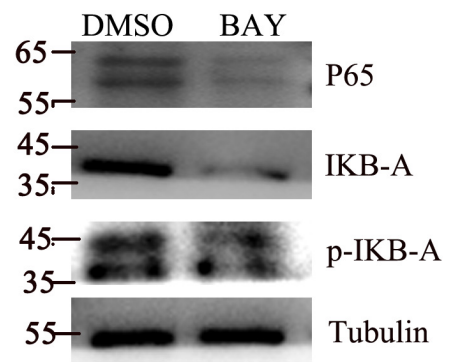

B
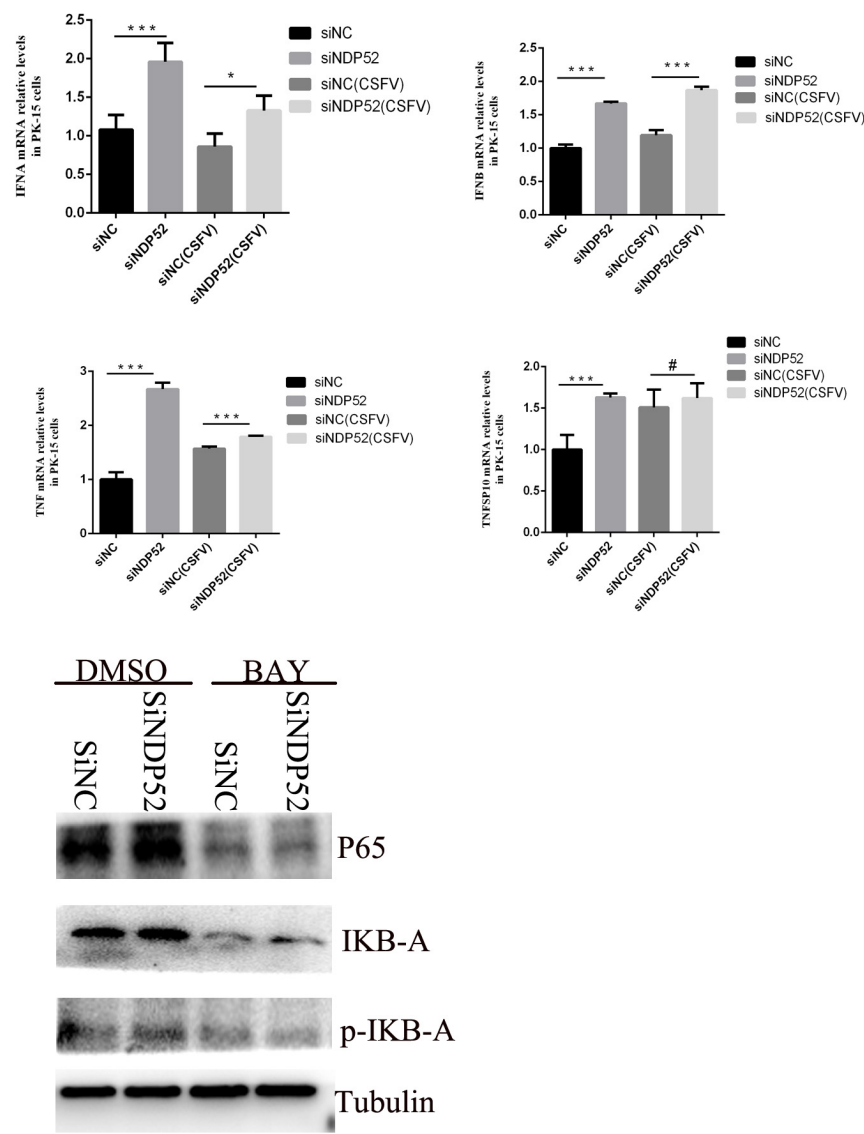

D
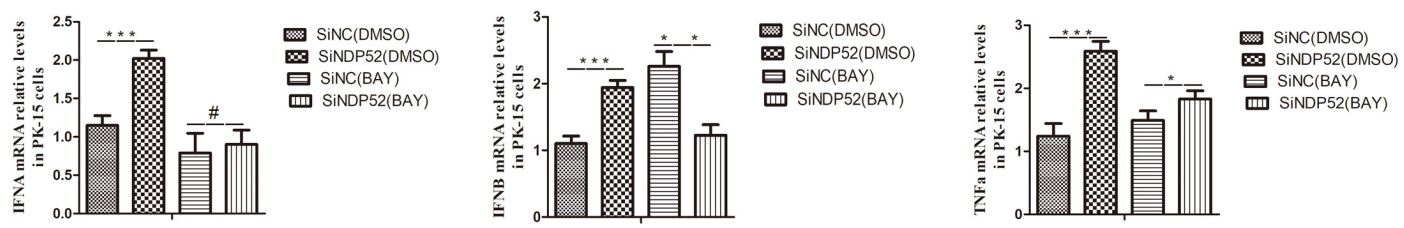

FIGURE 6 | Inhibition of NDP52 promotes innate immunity through the NF-kB signaling pathway. (A) Inhibition of NDP52 has a positive effect on NF-kB signaling pathway. PK-15 cells were mock-infected or infected with CSFV (MOI = 0.1) after transfection with siNDP52 for $24 \mathrm{~h}$. NF-kB signaling pathway proteins lkB-A, P65, IKB-Ras expression were analyzed by western blotting at $24 \mathrm{hpi}$. (B) PK-15 cells were treated as above. mRNA levels of the cytokines IFN- $\alpha$, IFN- $\beta$, and TNF were detected by qRT-PCR. (C) PK-15 cells were co-transfection with scrambled or NDP52 siRNA with DMSO or BAY (5 $\mu$ M) for $24 \mathrm{~h}$. NF-kB signaling pathway proteins IKB-A, P65, p-|кB-A expression were analyzed by western blotting at 24 hpi. (D) PK-15 cells were treated as (C), mRNA levels of the cytokines IFN- $\alpha$, IFN- $\beta$, and TNF were detected by qRT-PCR. The data represent mean \pm SD in three independent experiments. Data were tested by one-way ANOVA with post hoc LSD (L). ${ }^{*} P<0.05 ;{ }^{* *} P<0.01 ;{ }^{* *} P<0.001 ; \# P>0.05$.

Indeed, NDP52 inhibition also decreased LC3 expression and subsequent binding to $\mathrm{Ub}(\mathrm{A}-5)$ (Figure 4D).

\section{Inhibition of NDP52 Reduces Colocalization of Autophagy Vesicle CD63 With CSFV E2 Protein}

Our previous studies showed that CSFV induces autophagy, with autophagosomes as self-replication sites. However, the specific mechanism of CSFV-autophagosome entry is unclear (Pei et al., 2013). Because NDP52 regulates autophagy, we investigated the role of NDP52 in autophagic vesicles mediated by CSFV infection. We screened NDP52-interfering RNAs using western blotting and found that SiNDP52-1 had the best interference effect (Figure 5A). After NDP52 RNA interference for $24 \mathrm{~h}$, PK15 cells were infected with CSFV or mock-infected. Western blots showed that NDP52 inhibition decreased expression of CD63, a marker of autophagic vesicles (Figure 5B). Moreover, in the 


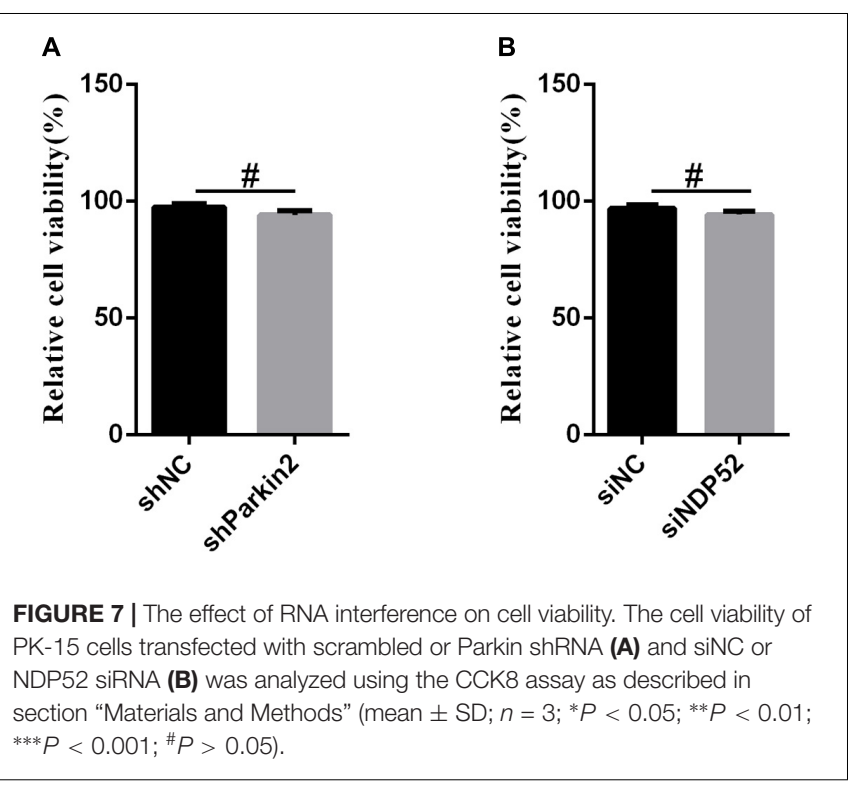

case of CSFV infection, this inhibition was more pronounced, indicating that NDP52 has a positive effect on the expression of CD63. To further verify the effect of NDP52 on CD63 and E2 colocalization, we used laser confocal microscopy to observe the localization of CD63. The results showed that CD63 binding to the CSFV E2 protein was decreased in the cytoplasm after NDP52 suppression (Figure 5C). We also found that Ub-5a binding to the CSFV E2 protein was reduced after NDP52 suppression (Figure 5D), suggesting that NDP52 plays an import role in CSFV entry into autophagosomes. These results indicate that NDP52 promotes colocalization of E2 with CD63 and $\mathrm{Ub}(\mathrm{A}-5)$.

\section{Inhibition of NDP52 Activates the NF-кB Pathway to Release IFN/TNF Cytokines}

NF- $\kappa \mathrm{B}$ activation during viral infection is part of the protective response to pathogens (Santoro et al., 2003). Interestingly, CSFV replication in cells suppresses type I IFN-inducible antiviral activity and apoptosis by interfering with IFN production, thereby resulting in the persistent survival of CSFV in host cells in vitro (Bensaude, 2004). To examine the effect of NDP52 on the innate antiviral immune pathway, we first examined NF- $\kappa B$ signaling activation following NDP52 inhibition. We observed increased expression of NF- $\kappa \mathrm{B}$ signaling pathway proteins after NDP52 inhibition, indicating that inhibition of NDP52 promotes the activation of the NF- $\mathrm{kB}$ signaling pathway (Figure 6A). We also found that inhibition of NDP52 promotes release of cytokines, including IFN- $\alpha$, IFN- $\beta$, and TNF (Figure 6B), indicating that NDP52 also plays an important role in the innate immune response. Therefore, NDP52 may inhibit CSFV infection via the NF- $\mathrm{KB}$ signaling pathway. In order to verify whether the release of SiNDP52 on the above cytokines is regulated by the NF- $\kappa \mathrm{B}$ pathway, we treated cells with NF- $\kappa \mathrm{B}$ inhibitor BAY 11$7082(5 \mu \mathrm{M})$ and found that BAY 11-7082 had no effect on cytokine release, but BAY 11-7082 affects the effect of SiNDP52 on IFN- $\alpha$ release (Figures 6 C,D).

\section{Cell Viability Was Not Affected by RNA Interference}

To exclude the possibility that NDP52 siRNA inhibited CSFV replication or that Parkin shRNA inhibited NDP52 protein modification by downregulating cell viability, we assessed the effects of RNA interference on the viability of PK-15 cells. The results showed no significant changes in the viability of cells following silencing of NDP52 or Parkin $(P>0.05)$ (Figure 7).

\section{DISCUSSION}

The body has several cellular immune mechanisms to maintain the internal environment and prevent invasion by pathogenic microorganisms. However, many viruses have evolved replication strategies to cope with the adverse host environment. Indeed, virus-induced autophagy as a protective mechanism has been widely reported (Sugden et al., 2005). In our previous study, we found that CSFV infection induces complete and high-yield autophagic flux and that CSFV replicates in the autophagosome. Together, these processes provide a suitable environment for long-term viral infection in host cells. Further research found that the viral E2 protein colocalizes with the autophagosome marker CD63 (Metzelaars et al., 1991), but the function of this interaction was unclear. As an autophagy receptor, NDP52 plays an important role both in bacterial and viral infection. NDP52 mediates targeting of cytosolic substrates to autophagy machinery, and promotes the maturation of autophagic vesicles (Von muhlinen et al., 2012; Cemma et al., 2014; Leymarie et al., 2017). Therefore, we wanted to define the role of the autophagy receptor NDP52 in CSFV infection. The current study provides further insights into the role of NDP52 in CSFVautophagic vesicle entry and the mechanism of induction of virus-mediated autophagy.

In the case of viral infection, Mohamud et al. (2018) reported that NDP52 binds to the coxsackievirus B3 viral protein VP1 and mediates VP1 ubiquitination via the K48 ubiquitin pathway. Other studies have shown that CALCOCO2 binds the nonstructural protein 2 of the Chikungunya virus to promote viral replication in humans (Judith et al., 2013), and plays a proviral role in measles viral infection (Petkova et al., 2017). In this study, our results showed that CSFV infection inhibits NDP52 expression. Moreover, NDP52-interfering RNA inhibits CSFV replication, indicating that NDP52 plays an important role in the infection of swine fever virus.

During viral infection, autophagy receptors can induce viral phagocytosis in a ubiquitin-dependent manner and can bind directly to viral capsid proteins or indirectly to host ubiquitin factors via E3 ubiquitin ligase (Mohamud et al., 2018). In the present study, we found that CSFV infection can reduce NDP52 ubiquitination and SUMO2-4 protein modification. Since the PINK1-Parkin pathway and autophagy receptors are closely related to functional ubiquitin modification, we examined the CSFV-mediated regulation of NDP52 protein modification after Parkin inhibition. CSFV can mediate NDP52 ubiquitination by PINK1-Parkin. Interestingly, when we inhibited NDP52 expression by RNA interference, 
we found that $\mathrm{Ub}(\mathrm{A}-5)$ expression increased while LC3 expression decreased. Ubiquitin acts as a signaling molecule, which causes polyubiquitination of autophagic substrates (Lu et al., 2017a,b). Autophagy receptor proteins recognize and bind autophagic substrates through a ubiquitin domain-dependent or independent manner (Walinda et al., 2014). The substrate is anchored to the autophagosome membrane by its LIR, followed by autophagosome-lysosome fusion and substrate degradation. We speculate polyubiquitination is required to recruit NDP52 or other autophagy receptors and reduce LC3 expression, and thus virus-induced ubiquitin molecules persist within the cell.

Because NDP52 mediates bacterial entry into autophagosomes (Sudhakar et al., 2019), we investigated whether NDP52 plays a role in CSFV entry into autophagosomes. We first assessed the effect of NDP52 on autophagy and observed that autophagy markers LC3 and Beclin-I decreased. Further analyses revealed that after NDP52 inhibition, CD63 protein expression was reduced, leading to decreased binding of CD63 to the viral E2 protein. These results suggest that NDP52 plays an important role in the mechanism of CSFV entry into autophagic vesicles. However, it is unclear whether NDP52 is modified by self-protein or by modifying CSFV protein to promote CSFV entry into autophagic vesicles.

NDP52 is involved in the negative regulation of the classical NF- $\kappa$ B pathway (Inomata et al., 2011). Our previous studies confirmed that CSFV can bind to MDA5 and RIG-1, inducing NF- $\kappa \mathrm{B}$ nuclear translocation via RIG-1 signaling (Xiao-Ying et al., 2013). Moreover, NF- $\kappa B$ signaling also regulates CSFV replication (Ling et al., 2018). However, the effect of NDP52 on the NF- $\mathrm{B}$ signaling pathway during CSFV infection was unclear. Therefore, we used RNA interference to inhibit NDP52 after CSFV infection. We found that NDP52 inhibition activates

\section{REFERENCES}

Becher, P., Avalos Ramirez, R., Orlich, M., Cedillo Rosales, S., König, M., Schweizer, M., et al. (2003). Genetic and antigenic characterization of novel pestivirus genotypes: implications for classification. Virology 311, 96-104. doi: 10.1016/ s0042-6822(03)00192-2

Bensaude, E. (2004). Classical swine fever virus induces proinflammatory cytokines and tissue factor expression and inhibits apoptosis and interferon synthesis during the establishment of long-term infection of porcine vascular endothelial cells. J. Gen. Virol. 85, 1029-1037. doi: 10.1099/vir.0.19637-0

Bizargity, P., and Schröppel, B. (2014). Autophagy: basic principles and relevance to transplant immunity. Am. J. Transplant. 14, 1731-1739. doi: 10.1111/ajt.12743

Cemma, M., Kim, P. K., and Brumell, J. H. (2014). The ubiquitin-binding adaptor proteins p62/SQSTM1 and NDP52 are recruited independently to bacteriaassociated microdomains to target Salmonella to the autophagy pathway. Autophagy 7, 341-345. doi: 10.4161/auto.7.3.14046

Ciechomska, I. A., Gabrusiewicz, K., Szczepankiewicz, A. A., and Kaminska, B. (2013). Endoplasmic reticulum stress triggers autophagy. Oncogene 32, 15181529. doi: $10.1038 /$ onc. 2012.174

Deretic, V., Saitoh, T., and Akira, S. (2013). Autophagy in infection, inflammation and immunity. Nat. Rev. Immunol. 13, 722-737. doi: 10.1038/nri3532

Fan, S., Yuan, J., Deng, S., Chen, Y., Xie, B., Wu, K., et al. (2018). Activation of Interleukin-1 $\beta$ Release by the Classical Swine Fever Virus Is Dependent on the NLRP3 Inflammasome, Which Affects Virus Growth in Monocytes. Front. Cell. Infect. Microbiol. 8:225. doi: 10.3389/fcimb.2018.00225

Fu, T., Liu, J., Wang, Y., Xie, X., Hu, S., and Pan, L. (2018). Mechanistic insights into the interactions of NAP1 with the SKICH domains of NDP52 and

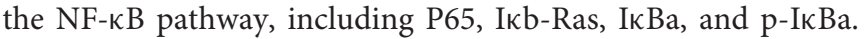
Furthermore, NDP52 inhibition promotes TNF and type I IFN release. CSFV infection attenuates this activation, suggesting that CSFV may have an inhibitory effect on the NF- $\mathrm{B}$ signaling pathway.

In summary, our study provides the first evidence that CSFV infection regulates and modifies the autophagy receptor NDP52. Further, we uncovered the dual CD63 and NF- $\mathrm{BB}$-induced mechanisms used by NDP52 to promote viral propagation.

\section{DATA AVAILABILITY STATEMENT}

All datasets generated for this study are included in the article/supplementary material.

\section{AUTHOR CONTRIBUTIONS}

SF, MiZ, KW, and JC conceived and designed the study. SF, KW, CL, MiZ, JC, EZ, and SM performed the experiments. SF, YC, MeZ, DS, and XL analyzed the data. SF, MiZ, KW, HD, JL, LY, and JC wrote the manuscript. All authors read and agreed upon the final manuscript.

\section{FUNDING}

This work was supported by grants from the National Natural Science Foundation of China (31672590), National Key Research and Development Program (2017YFD0501104 and 2016YFD0500600), and Science and Technology Program of Guangzhou, China (Nos. 2019B020211003 and 201803020005).

TAX1BP1. Proc. Natl. Acad. Sci. U.S.A. 115, E11651-E11660. doi: 10.1073/pnas. 1811421115

Gou, H., Zhao, M., Fan, S., Yuan, J., Liao, J., He, W., et al. (2017). Autophagy induces apoptosis and death of $\mathrm{T}$ lymphocytes in the spleen of pigs infected with CSFV. Sci. Rep. 7:13577. doi: 10.1038/s41598-017-14082-9

Heinz-Jurgen, T. R. S., Emilie, W., Tillmann, R., and Gregor, M. (1991). Hog cholera virus_molecular composition of virgins from a pestiferous. J. Virol. 65, 4705-4712.

Heo, J.-M., Ordureau, A., Paulo, J. A., Rinehart, J., and Harper, J. W. (2015). The PINK11-PARKIN mitochondrial ubiquitylation pathway drives a program of OPTN/NDP52 recruitment and TBK1 activation to promote mitophagy. Mol. Cell 60, 7-20. doi: 10.1016/j.molcel.2015.08.016

Hongchao, G., Mingqiu, Z., Hailuan, X., Jin, Y., Wencheng, H., Mengjiao, Z., et al. (2017). CSFV induced mitochondrial fission and mitophagy to inhibit apoptosis. Oncotarget 24, 39382-39400. doi: 10.18632/oncotarget.17030

Høyer-Hansen, M., and Jäättelä, M. (2008). Autophagy An emerging target for cancer therapy. Autophagy 4, 574-580. doi: 10.4161/auto.5921

Inomata, M., Niida, S., Shibata, K.-I., and Into, T. (2011). Regulation of Tolllike receptor signaling by NDP52-mediated selective autophagy is normally inactivated by A20. Cell. Mol. Life Sci. 69, 963-979. doi: 10.1007/s00018-0110819-y

Ivashkiv, L. B., and Donlin, L. T. (2013). Regulation of type I interferon responses. Nat. Rev. Immunol. 14, 36-49. doi: 10.1038/nri3581

Jin, S., Tian, S., Luo, M., Xie, W., Liu, T., Duan, T., et al. (2017). Tetherin suppresses type I interferon signaling by targeting MAVS for NDP52-mediated selective autophagic degradation in human cells. Mol. Cell 68, 308.e6-322.e6. doi: 10.1016/j.molcel.2017.09.005 
Johns, H. L., Bensaude, E., La Rocca, S. A., Seago, J., Charleston, B., Steinbach, F., et al. (2009). Classical swine fever virus infection protects aortic endothelial cells from pIpC-mediated apoptosis. J. Gen. Virol. 91, 1038-1046. doi: 10.1099/vir.0. 016576-0

Judith, D., Mostowy, S., Bourai, M., Gangneux, N., Lelek, M., Lucas-Hourani, M., et al. (2013). Species-specific impact of the autophagy machinery on Chikungunya virus infection. EMBO Rep. 14, 534-544. doi: 10.1038/embor. 2013.51

Kocaturk, N. M., and Gozuacik, D. (2018). Crosstalk between mammalian autophagy and the ubiquitin-proteasome system. Front. Cell Dev. Biol. 6:128. doi: 10.3389/fcell.2018.00128

Lazarou, M., Sliter, D. A., Kane, L. A., Sarraf, S. A., Wang, C., Burman, J. L., et al. (2015). The ubiquitin kinase PINK11 recruits autophagy receptors to induce mitophagy. Nature 524, 309-314. doi: 10.1038/nature14893

Leymarie, O., Meyer, L., Tafforeau, L., Lotteau, V., Costa, B. D., Delmas, B., et al. (2017). Influenza virus protein PB1-F2 interacts with CALCOCO2 (NDP52) to modulate innate immune response. J. Gen. Virol. 98, 1196-1208. doi: 10.1099/ jgv.0.000782

Ling, S., Luo, M., Jiang, S., Liu, J., Ding, C., Zhang, Q., et al. (2018). Cellular Hsp27 interacts with classical swine fever virus NS5A protein and negatively regulates viral replication by the NF- $\mathrm{kB}$ signaling pathway. Virology 518, 202-209. doi: 10.1016/j.virol.2018.02.020

Liu, T., Zhang, L., Joo, D., and Sun, S.-C. (2017). NF- $\kappa B$ signaling in inflammation. Signal Transduct. Target. Ther. 2:17023.

Liu, Z., Chen, P., Gao, H., Gu, Y., Yang, J., Peng, H., et al. (2014). Ubiquitylation of autophagy receptor optineurin by HACE1 activates selective autophagy for tumor suppression. Cancer Cell 26, 106-120. doi: 10.1016/j.ccr.2014.05.015

Lu, K., Den Brave, F., and Jentsch, S. (2017a). Pathway choice between proteasomal and autophagic degradation. Autophagy 13, 1799-1800. doi: 10.1080/15548627. 2017.1358851

Lu, K., Den Brave, F., and Jentsch, S. (2017b). Receptor oligomerization guides pathway choice between proteasomal and autophagic degradation. Nat. Cell Biol. 19, 732-739. doi: 10.1038/ncb3531

Lum, J. J., Bauer, D. E., Kong, M., Harris, M. H., Li, C., Lindsten, T., et al. (2005). Growth factor regulation of autophagy and cell survival in the absence of apoptosis. Cell 120, 237-248. doi: 10.1016/j.cell.2004.11.046

Majzoub, K., Wrensch, F., and Baumert, T. F. (2019). The innate antiviral response in animals: an evolutionary perspective from flagellates to humans. Viruses 11:758. doi: 10.3390/v11080758

Metzelaars, M. J., Wijngaard, P. L., Peter, P. J., Sixma, J. J., Nieuwenhuiss, H. K., and Cleversb, H. C. (1991). CD63 antigen. A novel lysosomal membrane glycoprotein, cloned by a screening procedure for intracellular antigens in eukaryotic cells. J. Biol. Chem. 266, 3239-3245.

Minowa-Nozawa, A., Nozawa, T., Okamoto-Furuta, K., Kohda, H., and Nakagawa, I. (2017). Rab35 GTPase recruits NDP52 to autophagy targets. EMBO J. 36, 2790-2807. doi: 10.15252/embj.201796463

Mohamud, Y., and Luo, H. (2018). The intertwined life cycles of enterovirus and autophagy. Virulence 10, 470-480. doi: 10.1080/21505594.2018.1551010

Mohamud, Y., Qu, J., Xue, Y. C., Liu, H., Deng, H., and Luo, H. (2018). CALCOCO2/NDP52 and SQSTM1/p62 differentially regulate coxsackievirus B3 propagation. Cell Death Differ. 26, 1062-1076. doi: 10.1038/s41418-0180185-5

Nakamura, S., and Yoshimori, T. (2017). New insights into autophagosomelysosome fusion. J. Cell Sci. 130, 1209-1216. doi: 10.1242/jcs.196352

Oeckinghaus, A., and Ghosh, S. (2009). The NF- B family of transcription factors and its regulation. Cold Spring Harb. Perspect. Biol. 1:a000034. doi: 10.1101/ cshperspect.a000034

Paton, D. J., and Greiser-Wilke, I. (2003). Classical swine fever-an update. Res. Vet. Sci. 75, 169-178. doi: 10.1016/s0034-5288(03)00076-6

Pei, J., Deng, J., Ye, Z., Wang, J., Gou, H., Liu, W., et al. (2016). Absence of autophagy promotes apoptosis by modulating the ROS-dependent RLR signaling pathway in classical swine fever virus-infected cells. Autophagy 12, 1738-1758. doi: 10.1080/15548627.2016.1196318

Pei, J., Zhao, M., Ye, Z., Gou, H., Wang, J., Yi, L., et al. (2013). Autophagy enhances the replication of classical swine fever virus in vitro. Autophagy 10, 93-110. doi: 10.4161 /auto. 26843

Petkova, D., Verlhac, P., Rozières, A., Baguet, J., Claviere, M., Kretz-Remy, C., et al. (2017). Distinct contributions of autophagy receptors in measles virus replication. Viruses 9:123. doi: 10.3390/v9050123
Ravenhill, B. J., Boyle, K. B., Von Muhlinen, N., Ellison, C. J., Masson, G. R., Otten, E. G., et al. (2019). The cargo receptor NDP52 initiates selective autophagy by recruiting the ULK complex to cytosol-invading bacteria. Mol. Cell 74, 320.e6-329.e6. doi: 10.1016/j.molcel.2019.01.041

Santoro, M. G., Rossi, A., and Amici, C. (2003). NF-кB and virus infection_who controls whom. EMBO J. 22, 2552-2560. doi: 10.1093/emboj/cdg267

Scherz-Shouval, R., Shvets, E., Fass, E., Shorer, H., Gil, L., and Elazar, Z. (2007). Reactive oxygen species are essential for autophagy and specifically regulate the activity of Atg. EMBO J. 26, 1749-1760. doi: 10.1038/sj.emboj.760 1623

Schmitz, M. L., Kracht, M., and Saul, V. V. (2014). The intricate interplay between RNA viruses and NF-кB. Biochim. Biophys. Acta Mol. Cell Res. 1843, 2754-2764. doi: 10.1016/j.bbamcr.2014.08.004

Shaid, S., Brandts, C. H., Serve, H., and Dikic, I. (2012). Ubiquitination and selective autophagy. Cell Death Differ. 20, 21-30. doi: 10.1038/cdd.2012.72

Sharma, V., Verma, S., Seranova, E., Sarkar, S., and Kumar, D. (2018). Selective autophagy and xenophagy in infection and disease. Front. Cell Dev. Biol. 6:147. doi: 10.3389/fcell.2018.00147

Sudhakar, P., Jacomin, A.-C., Hautefort, I., Samavedam, S., Fatemian, K., Ari, E., et al. (2019). Targeted interplay between bacterial pathogens and host autophagy. Autophagy 15, 1620-1633. doi: 10.1080/15548627.2019.1590519

Sugden, B., Jackson, W. T., Giddings, T. H., Taylor, M. P., Mulinyawe, S., Rabinovitch, M., et al. (2005). Subversion of cellular autophagosomal machinery by RNA viruses. PLoS Biol. 3:e156. doi: 10.1371/journal.pbio. 0030156

Tattoli, I., Sorbara, M. T., Vuckovic, D., Ling, A., Soares, F., Carneiro, L. A., et al. (2012). Amino acid starvation induced by invasive bacterial pathogens triggers an innate host defense program. Cell Host Microbe 11, 563-575. doi: 10.1016/j.chom.2012.04.012

Tumbarello, D. A., Waxse, B. J., Arden, S. D., Bright, N. A., Kendrick-Jones, J., and Buss, F. (2012). Autophagy receptors link myosin VI to autophagosomes to mediate Tom1-dependent autophagosome maturation and fusion with the lysosome. Nat. Cell Biol. 14, 1024-1035. doi: 10.1038/ncb2589

Verlhac, P., Grégoire, I. P., Azocar, O., Petkova, D. S., Baguet, J., Viret, C., et al. (2015a). Autophagy receptor NDP52 regulates pathogen-containing autophagosome maturation. Cell Host Microbe 17, 515-525. doi: 10.1016/j. chom.2015.02.008

Verlhac, P., Viret, C., and Faure, M. (2015b). Dual function of CALCOCO2/NDP52 during xenophagy. Autophagy 11, 965-966. doi: 10.1080/15548627.2015. 1046672

Von muhlinen, N., Akutsu, M., Ravenhill, B. J., Foeglein, A., Bloor, S., Rutherford, T. J., et al. (2012). LC3C, bound selectively by a noncanonical LIR motif in NDP52, is required for antibacterial autophagy. Mol. Cell 48, 329-342. doi: 10.1016/j.molcel.2012.08.024

Walinda, E., Morimoto, D., Sugase, K., Konuma, T., Tochio, H., and Shirakawa, M. (2014). Solution structure of the ubiquitin-associated (UBA) domain of human autophagy receptor NBR1 and its interaction with ubiquitin and polyubiquitin. J. Biol. Chem. 289, 13890-13902. doi: 10.1074/jbc.M114.555441

Wang, L., and Ou, J. J. (2018). Regulation of autophagy by Hepatitis C virus for its replication. DNA Cell Biol. 37, 287-290. doi: 10.1089/dna.2017.4115

Xiao-Ying, D., Wen-Jun, L., Ming-Qiu, Z., Jia-Ying, W., Jing-Jing, P., Yong-Wen, L., et al. (2013). Classical swine fever virus triggers RIG-I and MDA5-dependent signaling pathway to IRF-3 and NF- $\mathrm{KB}$ activation to promote secretion of interferon and inflammatory cytokines in porcine alveolar macrophages.pdf $>$. Virol. J. 10, 1-11.

Zaffagnini, G., and Martens, S. (2016). Mechanisms of selective autophagy. J. Mol. Biol. 428, 1714-1724. doi: 10.1016/j.jmb.2016.02.004

Conflict of Interest: The authors declare that the research was conducted in the absence of any commercial or financial relationships that could be construed as a potential conflict of interest.

Copyright (c) 2020 Fan, Wu, Luo, Li, Zhao, Song, Ma, Zhu, Chen, Ding, Yi, Li, Zhao and Chen. This is an open-access article distributed under the terms of the Creative Commons Attribution License (CC BY). The use, distribution or reproduction in other forums is permitted, provided the original author(s) and the copyright owner(s) are credited and that the original publication in this journal is cited, in accordance with accepted academic practice. No use, distribution or reproduction is permitted which does not comply with these terms. 